\title{
Pratiques
}

Linguistique, littérature, didactique

173-174| 2017

Le déjà-là dans l'écriture

\section{Dynamique de l'écriture et processus de resémantisation}

Dynamics of writing and resémantisation

\section{Sylvie Plane}

\section{(2) OpenEdition}

\section{Journals}

Édition électronique

URL : http://journals.openedition.org/pratiques/3307

DOI : $10.4000 /$ pratiques.3307

ISSN : 2425-2042

\section{Éditeur}

Centre de recherche sur les médiations (CREM)

\section{Référence électronique}

Sylvie Plane, «Dynamique de l'écriture et processus de resémantisation », Pratiques [En ligne] 173-174 | 2017, mis en ligne le 10 mars 2017, consulté le 01 mai 2019. URL : http:// journals.openedition.org/pratiques/3307 ; DOI : 10.4000/pratiques.3307

Ce document a été généré automatiquement le 1 mai 2019.

(c) Tous droits réservés 


\title{
Dynamique de l'écriture et processus de resémantisation
}

\author{
Dynamics of writing and resémantisation
}

Sylvie Plane

1 En 1935, à l'occasion d'un débat portant sur la mesure des états possibles de la matière selon la mécanique quantique, l'un des fondateurs de cette discipline, Erwin Shrödinger imagina une expérience mettant en scène un chat enfermé dans un caisson et susceptible d'être intoxiqué au moyen d'un mécanisme pervers et subtil. Dans cette expérience fictive mais fort célèbre - l'animal peut être considéré à la fois comme mort et comme vivant tant qu'un observateur n'a pas procédé à l'ouverture du caisson pour constater sa situation, laquelle ouverture anéantit irrémédiablement l'une des deux possibilités. Si ce protocole imaginaire a alimenté en son temps un débat entre physiciens au sujet de notions qui ne nous concernent guère ici, il constitue en revanche une sorte de représentation métaphorique du dilemme auquel se confronte l'analyste de l'écriture : si ce dernier se contente d'observer le texte produit, il se place en quelque sorte dans la situation du physicien qui découvre en ouvrant la boite mystérieuse l'état du chat sans pouvoir rétablir la chronologie des évènements; mais si, à l'inverse, il s'immisce dans le processus d'écriture pour traquer le scripteur, il perturbe ce processus comme le ferait l'expérimentateur qui entrouvrirait le caisson avant la fin de l'expérience.

2 Le présent article va se confronter à ce dilemme en s'interrogeant sur l'activité d'écriture à partir de la confrontation de positions théoriques contrastées et de l'analyse de données issues d'une expérimentation menée dans des classes de différents niveaux. L'objectif poursuivi ici sera de défendre l'hypothèse selon laquelle une partie de l'activité rédactionnelle consiste à recoder mentalement ce qui a été écrit, au fur et à mesure de la scription, et cela au prix d'une altération de certaines des propriétés linguistiques de la trace graphiée. L'angle d'attaque sera celui de la temporalité de l'écriture. On considèrera en effet l'écriture comme une activité inscrite dans une temporalité complexe puisque celle-ci concerne à la fois les opérations cognitivo-langagières mises en œuvre par le scripteur et le déploiement du texte au fur et à mesure de sa génération (Plane, Alamargot \& Lebrave, 2010). Cette approche de l'écriture sera étayée par les informations 
qu'on peut tirer de l'observation d'écrits d'élèves amenés à reproduire par écrit un récit qu'ils ont déjà transcrit une première fois. L'analyse des textes, dont quelques exemples seront présentés dans cet article, montre qu'il y a de grandes variations dans la manière dont s'opère la mémorisation et le traitement de son propre texte par un scripteur au cours de la production.

\section{Quels outils et quelles hypothèses pour rendre compte de la dynamique de la production d'écrit?}

3 Si pendant des siècles l'intérêt des théoriciens et des analystes s'est porté uniquement sur le produit de l'écriture - c'est-à-dire sur les textes - cela fait maintenant près d'un demisiècle que la production d'écrit est devenu un foyer d'attention. Cette recentration est concomitante avec le développement de travaux relevant de différents champs mais ayant en commun de prendre en considération la dimension temporelle de l'écriture, cette dimension étant la caractéristique majeure de toute dynamique.

\subsection{Des points de départs opposés pour décrire la temporalité de l'écriture}

La question des rapports entre temps et écriture est une question très ancienne qui a connu des développements divers. Dans les représentations collectives, l'écriture est sans conteste tout simplement associée à la stabilité, ce qui la met en quelque sorte à l'abri du temps et l'oppose à l'oral, lequel est ressenti - à juste titre - comme mouvant et fugace. En revanche, dans les analyses des linguistes la question de la temporalité est envisagée de manière plus subtile. Prenant comme point de départ l'étude des propriétés qu'ont les langues de figurer le temps au moyen de systèmes qui leur sont propres, les linguistes s'intéressent aux usages qu'en font les locuteurs-scripteurs pour rendre compte de leur propre positionnement vis-à-vis de l'objet de leur discours - voire de leur énonciation - et surtout pour conférer une épaisseur temporelle aux objets réels ou fictifs que leurs discours représentent, le récit étant, bien entendu, la forme discursive qui se prête le mieux à ce genre d'étude. Quasiment tous les courants de recherche en linguistique, de la psychomécanique à la linguistique textuelle et à la linguistique cognitive en passant par la pragmatique, de G. Guillaume (1993) à É. Benveniste (1966), H. Weinrich (1989), L. Gosselin (2006), C. Vetters (2004) et L. de Saussure (2003), pour ne citer qu'eux, ont exploré les relations entre les systèmes linguistiques, les mécanismes qu'ils sollicitent ou permettent chez les usagers de la langue, et le temps. Leurs préoccupations croisent celles de P. Ricœur (1983) qui fonde son approche phénoménologique du temps sur une analyse des rapports entre narration et mimésis. Linguistes et philosophes nous amènent donc à nous intéresser aux rapports entre temps et écriture à travers un questionnement sur la linéarisation de la substance linguistique, et par conséquent à nous interroger sur la temporalité de l'écriture, entendue comme acte graphique et scriptural.

5 Cette question de la temporalité de l'acte d'écriture a été traitée - sinon uniquement du moins de façon très majoritaire - dans deux secteurs de recherche, celui de la psychologie cognitive, d'une part, et celui de la critique génétique, d'autre part. Ces deux secteurs sont complémentaires, puisque l'un s'intéresse aux processus tandis que l'autre prend comme point de départ les textes, mais l'établissement de convergences requiert un 
délicat travail d'ajustement car les visées de ces domaines de recherche ainsi que leurs options théoriques - et par conséquent leurs méthodologies - accusent de fortes différences.

6 Dans le champ de la psychologie cognitive, l'angle d'attaque est celui de la modélisation des processus rédactionnels. En effet, depuis le modèle princeps de J. Hayes et L. Flower (1980) qui, en défendant l'idée de la récursivité des processus rédactionnels, avaient introduit une rupture avec les modèles rhétoriques étapistes qui dominaient jusqu'alors, l'objectif est désormais d'affiner les formalisations de l'activité scripturale en s'appuyant sur des expérimentations menées dans un cadre très contrôlé (voir notamment les synthèses de B. Bourdin en 1999, L. Chanquoy et D. Alamargot en 2002 et L. Heurley, en 2006). Cependant, si la plupart des modèles formels relevant de ce champ s'accordent désormais sur le fait que l'écriture requiert plusieurs niveaux de traitement (prélinguistique, linguistique, moteur, métacognitif), les questions de temporalité sont encore insuffisamment clarifiées et ne font pas consensus. Or, leur clarification est un enjeu pour ces modèles dans la mesure où leur pertinence dépend de leur capacité à établir l'enchainement des processus cognitifs et leurs interactions, comme l'ont montré A. Piolat et J.-Y. Roussey (1992). Plus précisément, les divergences concernent les fonctionnements mnésiques, avec deux conceptions qui s'opposent, celle issue du modèle capacitaire de la mémoire de travail (MdT) développé par A. Baddeley (1986) et appliqué à la rédaction par D. McKutchen (1998) et celle de la mémoire de travail à long terme (DdTLT) développée par R. T. Kellogg (1999). Le modèle de R. T. Kellog a pour spécificité de s'attacher à rendre compte de l'administration des sous-processus, et en particulier du calepin visuo-spatial impliqué dans la production d'écrit.

7 L'évolution des modèles de production est fortement liée à l'évolution des technologies de recueil de données en temps réel qui se sont donné pour finalité de fournir des observables permettant au chercheur de reconstituer par inférence les opérations cognitives réalisées par les scripteurs. On est passé du simple enregistrement chronométrique de l'alternance entre activité graphique et pauses, tel que le faisaient A. Matsuhashi (1981) ou G. Fortier et C. Préfontaine (1994), à des dispositifs plus sophistiqués de suivi de la frappe sur ordinateur soit lors de tâches très ciblées (Kellogg, Turner, Whiteford \& Mertens 2016), soit lors de production de textes avec notamment Inputlog développé par M. Leijten et L.van Waes (2006) et Scriptlog, utilisé par S. Strömqvist et al. dans une perspective développementale (2006). L'écriture manuscrite est observée quant à elle par des dispositifs d'eye tracking qui permettent d'établir des corrélations entre les mouvements oculaires et les gestes graphiques, tel Eye and pen, developpé par $\mathrm{D}$. Alamargot et $\mathrm{D}$. Chenet ou d'établir des graphes caractérisant des modes de scription comme le font C. Leblay et G. Caporossi (2014).

8 Dans le champ de la critique génétique, contrairement à la perspective qui guide les recherches en psychologie cognitive, il ne s'agit nullement d'aboutir à une modélisation mais au contraire de caractériser un objet d'étude toujours singulier. L'objectif est en effet de reconstituer la genèse d'un texte particulier, comme l'ont fait 0 . Lumbroso (2004) ou P. Hamon (2009, p. 15) à partir des carnets d'É. Zola, ou de définir le mode d'écriture d'un auteur en se fondant sur l'observation de régularités dans les opérations d'écriture, comme le propose S. Pétillon (2006). En effet, comme l'a montré A. Grésillon (1994), la chronologie de l'écriture de textes littéraires ou spéculatifs, uniques par essence, peut être établie à partir des informations fournies par les dossiers préparatoires constitués par les auteurs et du repérage des opérations d'écriture. Mais, comme l'explique L. Hay 
(1979), contrairement à la philologie classique, l'établissement de cette chronologie n'est pas soumis à une conception téléologique de l'écriture qui voudrait que les interventions successives de l'auteur sur son texte constituassent nécessairement des améliorations. Les analyses visent plutôt à rendre compte des stratégies d'écriture, conscientes ou non, dénoncées par la succession des opérations et par les stratifications énonciatives qui informent sur le dialogue virtuel entre le texte et son auteur.

Pour reprendre la métaphore évoquée plus haut, on pourrait dire, de façon un peu réductrice, que la psychologie cognitive dans son désir de suivre au plus près le chat de Shrödinger l'accompagne mais réduit ainsi son espace de liberté, tandis que la génétique textuelle attend que l'animal soit à sa disposition pour tenter de savoir ce qui lui est arrivé.

\subsection{Une dynamique qui résiste à l'observation}

Entre ces deux approches des ponts ont été jetés, comme le signale L. Hay (2016). Le dialogue a permis que soient mises en place des méthodologies empruntant aux deux champs, en particulier celles qui portent sur le temps réel, et que des outils d'analyse circulent d'une communauté scientifique à l'autre, comme en témoignent notamment les publications interdisciplinaires dirigées par S. Pétillon \& F. Ganier (2006) et par I. Fenoglio \& L. Chanquoy (2007). Des convergences ont été facilitées par la construction d'un cadre commun élaboré par S. Plane, T. Olive et D. Alamargot (2010) qui définit l'activité de production d'écrit comme le traitement d'un système de contraintes de deux ordres, linguistiques et psycholinguistiques, antagonistes, non hiérarchisables entre elles, et offrant des degrés de liberté différents. La complexité des contraintes linguistiques et la variabilité du degré de liberté qu'elles laissent au scripteur rendent impossible le recours à une résolution préprogrammée. En effet, comme l'a montré H. Nolke (1999), en raison de la diversité des composantes entrant en jeu dans les phénomènes linguistiques et de la spécificité de leurs règles internes, il n'existe pas d'algorithme de résolution qui permettrait d'automatiser les choix linguistiques lors de la production verbale. On retrouve la même complexité du côté des contraintes psycholinguistiques, car leur gestion sollicite la mémoire de travail qui doit simultanément opérer des traitements langagiers et stocker de l'information (Kellogg, Olive \& Piolat, 2007); or la capacité de cette mémoire est limitée, ce qui pèse sur la mise en œuvre des processus impliqués dans la rédaction comme l'ont montré notamment M. Fayol (1999, p. 49) et M. Fayol et P. Largy (1992) à l'occasion de protocoles de double tâche. Les pauses en cours d'écriture et les erreurs (fautes de frappe ou erreurs graphiques, erreurs orthographiques, lapsus calami, etc.) sont autant d'indices qui signalent que l'attention du scripteur a été mobilisée sur un aspect de la production d'écrit au détriment d'un autre en raison de la multiplicité des contraintes à gérer simultanément.

11 Le temps n'est donc pas simplement un élément du contexte de la production d'écrit, mais un facteur qui joue un rôle à deux niveaux et inscrit l'écriture dans une dynamique complexe. En effet, toute production de texte met en jeu simultanément deux processus, l'activité cognitivo-langagière du scripteur et la génération du texte, or ces deux processus sont interdépendants mais imparfaitement synchrones. Le fait que le développement du texte dépende de l'activité du scripteur n'a guère besoin d'être démontré, mais quelques mots sont nécessaires pour montrer que la relation de dépendance est réciproque. Il se trouve en effet que les actes décisionnels du scripteur 
sont en partie déterminés par le substrat sur lequel ils s'exercent, c'est-à-dire par le texte, or l'état de ce substrat change continument, puisqu'en s'incrémentant au fur et à mesure de sa scription il passe, grâce à une suite continue d'étapes, d'une image virtuelle floue et sans contour qu'est le projet initial du scripteur à un texte effectif. Le travail cognitif du scripteur s'appuie donc sur un objet évolutif, le texte en cours d'écriture ; or l'évolution de cet objet altère les degrés de liberté offerts au scripteur : la partie de texte déjà écrite constitue en quelque sorte une consigne, comme dans l'exercice scolaire de la suite de texte où un incipit impose ses règles au texte à venir, si ce n'est que cet incipit s'incrémente constamment et fait donc évoluer les consignes d'écriture. Cette restriction de l'espace de liberté laissée à l'auteur au fur et à mesure de la production de son texte est évoquée par de nombreux auteurs qui témoignent à cette occasion des rapports ambigus qu'ils entretiennent avec leur œuvre. C'est le cas notamment du scénariste de bédé Alain Ayroles $^{1}$ qui, dans un entretien où il présente sa méthode de travail, explique comment les possibilités d'improviser se réduisent au fur et à mesure de l'avancée dans l'écriture de récit.

12 La tâche pour le scripteur est rendue complexe dès lors que le texte atteint une certaine longueur car celui-ci n'est plus immédiatement disponible en mémoire de travail et doit faire l'objet d'une récupération en mémoire à long terme et/ou d'une consultation directe. Plusieurs indices informent sur les aléas de la récupération en mémoire. Ainsi il arrive que soient commises des erreurs qui attestent que le scripteur s'est appuyé, par exemple pour gérer des questions de cohérence ou d'accords grammaticaux, sur des segments de texte qui avaient été graphiés mais qui ont été ultérieurement supprimés, notamment par effacement dans l'écriture informatique. Ainsi s'expliquent certaines fautes d'accord qui procèdent d'une relation syntagmatique entre le segment linguistique qu'on ajoute et un segment disparu mais conservé en mémoire, comme si le scripteur prenait appui sur un membre fantôme. Le recours à la consultation visuelle du texte en cours d'écriture est attesté quant à lui par les expérimentations utilisant des dispositifs de suivi des mouvements oculaires. D. Alamargot, S. Plane, E. Lambert \& D. Chesnet (2009) ont même montré que les modalités du report visuel en cours de scription sur le texte déjà produit différaient en fonction de l'expertise des scripteurs.

Plus exactement, au fur et à mesure qu'il écrit, l'auteur d'un texte est amené à opérer deux grands types de traitements mnésiques de son propre texte afin de pouvoir s'y référer, traitements qui concernent les aspects graphiques - ou plus exactement matériels - et sémantiques du texte : i) le scripteur établit de façon non consciente des repères topologiques en stabilisant le souvenir de la disposition spatiale de son texte, ce qui lui permet de se reporter visuellement en cours d'écriture à tel ou tel passage pour le vérifier ou le consulter, comme l'ont montré N. Le Bigot, J.-M. Passerault et T. Olive (2011) ; ii) il élabore et enregistre une image du contenu sémantique et des propriétés linguistiques du texte. En d'autres termes, il ne mémorise pas son texte tel quel mais procède sur son texte à un ensemble d'opérations que je propose d'appeler resémantisation , pour des raisons que j'expliquerai, et qui s'apparentent à celles à l'œuvre dans l'activité de lecture et dans celle de production verbale orale. 


\subsection{Production d'écrit et resémantisation : la production verbale écrite, entre lecture et production verbale orale}

W. Kintsch et T. A. van Dijk (cf. synthèse dans G. Denhière \& S. Baudet, 1987) qui ont défini les principes de l'analyse propositionnelle à l'origine de la base de texte, il est généralement admis que lors de la tâche de lecture/compréhension, l'activité cognitive du lecteur est orientée non vers la mémorisation fidèle de chaque segment linguistique mais vers la représentation du contenu. Le traitement en réception des matériaux linguistiques est en effet mis au service de l'élaboration d'une structure délinéralisée au sein de laquelle les informations fournies par le texte lu sont interreliées. J.-F. Le Ny (1989, p. 192) signale également qu'en raison des phénomènes d'inférence intervenant au cours de la lecture, la source d'une information peut ne pas avoir été mémorisée par le lecteur. Ainsi, à la fin de la lecture d'un récit, le lecteur peut en reformuler la diégèse et citer de mémoire des formules extraites du texte, mais il ne peut pas restituer le texte mot à mot. En cela l'activité de lecture-compréhension s'oppose à celle d'apprentissage par cœur, activité dont la visée première est non pas de comprendre mais de stocker in extenso le texte. particularité le fait que le locuteur doit faire un effort de mémorisation ayant pour objet ses propres propos. Ainsi ce que J.-M. Barbéris et B. Maurer (1998) appellent « ratage » et toutes les scories de l'oral signalent-ils non seulement la complexité de la tâche d'énonciation mais aussi la difficulté de la mémorisation d'un texte oral par son auteur. Cela se constate aisément dans des situations ordinaires de communication : les limites de la mémoire de travail interdisent à un locuteur de réitérer in extenso l'énoncé qu'il vient lui-même de produire pour peu que cet énoncé soit long, ce qui explique d'ailleurs certaines des particularités structurelles des énoncés oraux.

16 Les situations d'écriture, de lecture et de production verbale orale ont donc toutes les trois en commun d'exiger que le sujet réalise en cours d'activité des opérations de stockage d'information. Mais dans le cas de la lecture, les éléments à mémoriser sont issus d'un texte produit par un agent extérieur, l'auteur, alors que dans le cas de l'écriture et de la production orale, les éléments à mémoriser sont issus du discours produit par le sujet lui-même. En revanche, lecture et production d'écrit offrent toutes deux la possibilité de se reporter visuellement aux données sources et donc de les consulter.

17 La production d'écrit présente donc les similitudes et les différences avec la lecture et la production orale évoquées plus haut; mais elle comporte en outre deux spécificités notables : d'une part, le texte à partir duquel s'opère la mémorisation a été rédigé par le scripteur et est donc composé d'éléments langagiers que le scripteur avait lui-même déjà mémorisés précédemment sous des formes plus ou moins abouties; d'autre part, ce texte a été en partie stabilisé, même de façon provisoire et réversible, par l'acte graphique. Cependant, comme il a été dit plus haut, le texte produit n'est pas mémorisé tel quel in extenso par son auteur, comme le montre le fait que celui-ci le consulte constamment en cours de scription. Ces considérations plaident en faveur de l'hypothèse selon laquelle l'ensemble des opérations mises en œuvre par un auteur lors de la mise en mémoire de son texte, au cours de l'activité d'écriture ou postérieurement, constitue une véritable transformation: la substance sémantique est en partie conservée, mais de nouvelles 
hiérarchies informationnelles se mettent en place, altérant les liens internes qui structuraient cette substance et provoquant l'émergence de nouveaux réseaux de signifiants. J'avais envisagé d'intituler «recodage» ce processus, mais ce terme ne m'a paru apte à rendre compte des transformations opérées, car il dénote un ensemble d'opérations qui peuvent s'apparenter à la traduction, laquelle vise à la conservation intégrale du signifié et à sa restitution au moyen d'un autre système linguistique. Or les opérations qui sont en jeu dans l'écriture sont plus complexes que cela: des effets de saillance, ou au contraire des effacements, des coalescences, des dislocations peuvent se produire et être à l'origine d'un fantôme de texte mémorisé qui a ses propres accentuations, bien différentes de celles du texte qui a été réellement écrit, d'où le terme de « resémantisation » qui m'a paru plus à même d'évoquer cette reconstruction. Il va de soi que le phénomène de distorsion entre le texte écrit et l'image de ce texte est d'autant plus accentué que texte est long ou que son écriture s'étale dans le temps.

Pour mettre à l'épreuve cette hypothèse, une expérimentation a été mise en place. Elle a eu pour objectif de mettre au jour le processus de resémantisation à travers le repérage de ses effets ainsi que les variantes interindividuelles dans sa mise en œuvre par des élèves, à partir de l'observation des altérations entre deux versions d'un même texte, dont la première était issue d'un substrat fourni à ces élèves.

\section{Un dispositif pour observer les traces de resémantisation}

\subsection{Contraintes méthodologiques}

L'expérimentation dont quelques éléments vont être présentés a été mise en place dans une perspective didactique même si ce n'est pas cet aspect-là qui sera mis en avant dans cet article ${ }^{2}$. Il s'agissait en effet de caractériser les modes d'écriture usités par des enfants d'âge scolaire afin d'aider à la mise en place de situations d'enseignement appropriées. Pour l'organisation de cette expérimentation, trois impératifs ont été pris en compte :

- respect des situations écologiques. Cela impose que l'expérimentation se déroule dans le cadre ordinaire des classes en y apportant le moins de perturbation possible et avec la collaboration des enseignants. Ces conditions interdisent donc le recours à des matériels sophistiqués tels que les dispositifs de suivi oculaire et conduisent à proposer des activités proches de celles auxquelles les élèves sont habitués. Stricto sensu, il s'agit donc d'un protocole quasi expérimental car, contrairement aux situations de laboratoire, les expérimentations en classe mettent en jeu des faisceaux de paramètres - conditions matérielles, contexte pédagogique etc. - qu'il est impossible de neutraliser ou d'unifier.

- collecte d'éléments permettant des comparaisons étroites entre les procédés employés par les différents scripteurs. Pour cela, il fallait éviter que ces opérations interviennent sur des substrats hétérogènes, ce qui aurait été le cas si la consigne d'écriture avait laissé aux scripteurs la liberté d'inventer de toutes pièces le texte qu'ils allaient écrire. Pour juguler le risque de disparité, il a été choisi d'imposer comme point de départ aux scripteurs participant à l'expérimentation un même substrat initial, à savoir un conte ; et pour disposer d'un point de repère aisé à exploiter, le même conte a été présenté sous deux formes différant entre elles par un seul point, le mode de discours rapporté.

- neutralisation des difficultés de déchiffrage : si au cours de la première étape il avait été demandé aux élèves de lire un texte, il était à craindre que les lecteurs malhabiles 
rencontrent des difficultés de compréhension en raison de leur maitrise insuffisante de la lecture. Pour cette raison, le point de départ de l'expérimentation a été un texte communiqué oralement aux élèves et non donné à lire.

\subsection{Le texte source}

Le support de l'expérimentation a pour origine un conte que Raponda Walker a collecté et traduit de l'omyéné en français. Ce conte, Les trois ruses de la gazelle, a l'avantage de présenter des similitudes structurelles avec les contes auxquels les élèves scolarisés en France sont habitués, avec une triplication qui en facilite la mémorisation. Il participe de la même veine que le Roman de Renart en mettant en scène un animal rusé, la gazelle, qui trompe un animal plus fort que lui, le léopard. Mais il a aussi l'avantage inverse de ne pas faire partie du répertoire culturel classique des élèves car il se situe dans un univers exotique : il y est question de vin de palme, d'une boisson nommée itoutou, d'antilopes et de filets pour transporter les proies. Nous avons adapté le texte de façon à lisser les principales difficultés lexicales et avons élaboré deux versions de ce texte qui ne diffèrent entre elles que par la présentation des paroles de personnages : dans l'une les propos des personnages sont rapportés au discours direct (texte A), dans l'autre le discours direct est évité (texte B). La restitution du discours rapporté par les élèves a fait l'objet d'une analyse (Plane, Rondelli \& Vénérin, 2013) et les choix en matière de lexique sont examinés par C. Vénérin dans ce numéro de Pratiques.

\subsection{Déroulement}

Le dispositif a comporté quatre phases :

\section{a) Audition par les élèves du texte du conte intitulé Les trois ruses de la gazelle}

Le texte, qui avait été enregistré par un lecteur ayant consigne de ne pas marquer d'effet d'accentuation, a été diffusé aux élèves. La transmission par le biais d'un enregistrement a permis d'éviter les disparités introduites par la manière dont chaque enseignant aurait interprété le texte s'il avait dû lui-même assurer la lecture orale.

- Durée de l'enregistrement : texte A : 5'30"; texte B : $5^{\prime} 28^{\prime \prime}$

- Nombre de mots graphiques : texte A : 1050 ; texte B : 989 .

\section{b) Restitution par écrit du texte entendu, immédiatement après audition}

Les élèves avaient reçu la consigne d'écouter attentivement le conte enregistré, de ne prendre aucune note et de s'efforcer de le mémoriser car il leur serait demandé de le mettre par écrit sitôt après l'audition. Ils ont été avertis que dans l'analyse que l'on ferait de leurs textes, il ne serait pas tenu compte de l'orthographe, ceci pour éviter les contournements auxquels procèdent bon nombre d'élèves face aux difficultés orthographiques lors de tâches de rédaction. 
comme entre l'audition et le premier rappel, les élèves n'ont eu accès ni à leurs textes ni à l'enregistrement du conte.

\subsection{Les données collectées}

Onze classes étaient concernées, totalisant 245 élèves du CM à la classe de seconde. $\mathrm{Ne}$ seront évoqués ici que les résultats concernant le rappel de récit immédiat et le rappel différé.

31 Nous disposons de deux corpus copieux, le corpus A composé des productions écrites de 104 élèves ayant eu comme point de départ le texte avec discours direct, le corpus B des productions de 141 élèves ayant eu l'autre texte comme point de départ.

\section{Aperçu sur les traces relevées: quelques faits saillants attestant de la diversité des procédures}

Les données recueillies sont abondantes et donnent un aperçu de la diversité des tendances qui s'opposent, fournissant ainsi des éléments pour établir des catégories susceptibles d'aider à définir des styles de scription.

\subsection{Du texte source au rappel de récit : focalisation du scripteur sur la schématisation vs focalisation sur la forme linguistique}

La première version des textes recueillis est toujours d'une longueur inférieure à celle du texte source. Ainsi alors que le point de départ était un texte de 989 mots graphiques, la première version la plus longue produite par un élève est un écrit de 543 mots graphiques. Il n'y a rien d'étonnant à ce que le rappel de récit soit plus court que le texte source, car d'une part, les limites des capacités mémorielles font qu'il est impossible de retenir dans sa complétude un texte long, d'autre part, la tâche de compréhension que s'imposent implicitement les auditeurs/lecteurs d'un récit met en œuvre une sélection des informations fournies par le texte source en vue de leur intégration.

Mais il est intéressant de noter que deux tendances opposées se manifestent - avec tous les gradients possibles entre les deux pôles - avec d'un côté les textes dans lesquels la réduction procède majoritairement de la sélection de formules issues du texte source que le scripteur agence entre elles, et à l'opposé, des textes privilégiant la reformulation synthétique. Ou pour reprendre la typologie que J.-B. Grize (1992) appliquait aux résumés, pour certains scripteurs, la mémorisation du texte s'apparente au " résumé-échantillon ", tandis que pour d'autres elle se rapproche de la synthèse en privilégiant les opérations de schématisation et la reformulation.

Les incipits sont particulièrement éclairants à ce sujet. Ainsi on comparera ${ }^{3}$ :

(1) La Gazelle, qui avait emprunté une grosse somme d'argent au Léopard, retardait de jour en jour le moment de rembourser cet argent. Fatigué d'attendre, le Léopard décida un jour d'aller réclamer son argent à la Gazelle (Texte source B)

(2) La gazelle avait emprunté une somme d'argent au léopard. Le léopard fatigué d'attendre décida d'aller chercher l'argent. (Jé 6v1)

(3) Un léopard donne de l'argent à une gazelle. Mais ensuite il souhaite récupérer cette argent. (Mo 2v1) 

évoqué le récit entendu. Cette éventualité est incontrôlable, mais le fait que la première passation n'ait pas été suivie d'un temps qui aurait permis aux élèves de discuter entre eux du texte qu'ils venaient d'écrire fragilise cette hypothèse : il est probable qu'elle ne peut s'appliquer à l'ensemble des élèves dont la deuxième version est plus étoffée que la première.

41 ii) lors de la première épreuve de rappel, l'effort cognitif exigé par la textualisation n'aurait pas permis d'allouer suffisamment de ressource à la remémoration d'éléments stockés en mémoire à long terme. En revanche, lors de la deuxième épreuve de rappel, la tâche de textualisation serait plus aisée car elle a déjà été effectuée une première fois et fixée par sa transcription graphique, ce qui offre au scripteur plus de disponibilité pour mobiliser ses souvenirs du récit entendu $u^{4}$. La différence de fluidité graphique entre les deux versions est un indice en faveur de cette interprétation. Ainsi la première version de Guillaume, élève de seconde, comporte 48 mots + 9 mots barrés + 1 ébauche de mot, alors que la version issue du rappel différé, d'une graphie plus maitrisée, en comporte 231 et uniquement deux ratures.

iii) entre les deux passations, le texte continue à travailler son auteur, pour reprendre le mot de Ph. Lejeune (2005) qui expose comment « [l'] écriture déborde le moment où l'on écrit ». I. Fenoglio (2007) parle de la «rumination» du texte, D. Bucheton (1996) de son "épaississement », l'une et l'autre signalant que les traces graphiques observables (y 
compris celles figurant dans les avant-textes) ne renseignent que très imparfaitement sur les contours temporels de la production textuelle. Autrement dit, la production textuelle proprement dite n'est qu'un fragment découpé par l'artefact que constitue la scription dans une activité langagière continue qui commence avant même l'acte d'écrire, avec ce qu'U. Eco (1992) appelle les intentiones auctoris et se prolonge par le ressassement assumé ou subi - du texte produit. Les éléments qui vont suivre constituent des arguments en faveur de cette hypothèse.

\subsection{Du texte source aux deux versions : développement des scripts et jeu de l'intertextualité}

S'il est vrai que, comme on s'y attend, on note souvent une perte de substance d'une version à l'autre, il arrive cependant que l'on constate aussi l'inverse. L'apport d'informations supplémentaires a été relevé aux différents stades du dispositif : le texte issu du rappel immédiat peut contenir dans certains passages plus d'informations que le texte source, et la seconde version peut à son tour être plus explicite ou précise que la première.

La comparaison des différents états de texte (texte source/version 1/version 2) montre que cet enrichissement est principalement dû à deux procédés qu'il est parfois difficile de dissocier : le développement d'un script et la mobilisation de références intertextuelles.

Les scripts - ou pour J.-F. le Ny (1989, p. 130) les scénarios - ont surtout été étudiés dans les travaux portant sur la lecture. Il a été montré depuis longtemps que la mémorisation de scripts partagés culturellement, c'est-à-dire de suites d'actions ordonnées dans le temps et procédant de la décomposition analytique de situations ordinaires, est un instrument de la compréhension. Ces scripts, qui font partie de l'encyclopédie personnelle du lecteur sont convoqués notamment pour résoudre par inférence des problèmes de référence et combler, comme le dit U. Eco $(1985$, p. 29), les vides de cette machine paresseuse qu'est le texte. Du côté de la production d'écrit, ils ont été étudiés à travers les stéréotypes que mobilisent les apprentis scripteurs, notamment par B. Kervyn et J.-L. Dufays (2003).

Des exemples comme le suivant donnent à penser que les scripts interviennent non seulement dans la lecture et dans l'inventio lors de la conception d'un texte, mais également dans le processus de mémorisation de son propre texte par le scripteur.

Le texte source se présente ainsi :

(4) Il arriva chez la Gazelle, tandis qu'elle était encore au lit. La Gazelle, gênée par

l'arrivée subite du Léopard, lui fit cependant bon accueil [...]

La première version de l'élève reprend une partie du lexique initial :

(5) Il arriva chez la gazelle a une heure bien matinal, mais elle l'accueilla malgré tout assez chaleureusement. (Ev 6v1)

La forme est plus condensée mais comporte cependant l'ajout d'une précision horaire ( a une heure bien matinal ») issue de l'interprétation de le situation narrée.

La seconde version quant à elle ne fait plus mention des circonstances temporelles mais développe un script :

(6) Un jour, il décida d'allé lui réclamé, il arriva devant la porte et toqua. La gazelle

ouvrit la porte. (Ev 6v2) 
51 très probable que ce script de l'introduction dans un domicile est issu de la lecture de contes et fait partie du répertoire des jeunes lecteurs, car nous trouvons dans les textes d'un autre élève, scolarisé dans un autre établissement et donc sans contact avec le premier, un développement analogue :

(7) La gazelle le vit arriver et fit comme si elle ne l'avais pas vu. Le léopard toca.

(Ra6v1)

La présence de ces scripts ou fragments de scripts est un élément qui corrobore les résultats des recherches menées dans une perspective bakhtinienne qui ont montré l'importance de la caractérisation générique dans la production de texte par des jeunes scripteurs, et notamment les travaux de B. Lafourcade (2008). Dans notre corpus, le genre du conte avait nettement été identifié comme le prouve l'abondance des commentaires métadiscursifs (" c'est l'histoire de ») et des marques caractérisant le conte (« il était une fois ») relevées dans les textes produits. Or s'il est acquis que lors de l'inventio, le texte puise une partie de ses traits dans les ressources génériques dont dispose le scripteur, les constats que nous avons faits nous amènent à ajouter que ce phénomène se prolonge lors de la mise en mémoire du texte par son auteur. En quelque sorte, lors de la mise en mémoire du texte, qui intervient tout au long de sa production, ce texte s'incorpore progressivement au patrimoine interne du scripteur et y puise en retour de nouveaux traits, ce qui fait que lorsque le scripteur cherche à se remémorer ce qu'il a écrit, il peut convoquer, par contagion, des éléments issus d'autres productions langagières proches.

\section{Des pistes de travail en guise de conclusion}

Il reste encore beaucoup d'analyses à faire pour cerner de plus près la manière dont s'opère la resémantisation en cours d'écriture. J'avais déjà noté dans un article de 2003, qu'il arrivait que des scripteurs se comportent vis-à-vis de leur de leur propre écrit comme s'il s'agissait d'un texte qui leur serait étranger et ne parviennent plus à adhérer au système diégétique qu'ils avaient eux-mêmes mis en place. Ce que j'avais constaté alors m'apparaissait comme une sorte de désaffection de la part du scripteur qui ne parvenait plus à être le lecteur coopérant de son propre texte. Il me semble aujourd'hui que l'hypothèse de la resémantisation en cours d'écriture pourrait rendre compte de ce phénomène. Il est très probable qu'il existe de fortes variables dans la sélection et la mise en œuvre des procédures de resémantisation. Certaines doivent être en lien avec l'âge des scripteurs notamment en raison des rôles que jouent la fluence de l'écriture et la composition des ressources langagières stockées sous la forme de schémas et de ce que M.-A. Paveau $(2006,2007)$ appelle les prédiscours. Mais ces facteurs ne suffisent pas à rendre compte de la diversité des procédures et il reste à identifier d'autres variables, l'ambition n'étant pas de catégoriser les scripteurs mais au contraire de mettre en évidence des fonctionnements idiosyncrasiques qui témoignent des différentes façons d'être auteur de son texte. 


\section{BIBLIOGRAPHIE}

alamargot, D., Plane, S., LAmbert, E. \& Chesnet, D. (2010). « Using Eye and Pen Movements to Trace the Development of Writing Expertise: Case Studies of a Seventh, Ninth and Twelfth Grader, Graduate Student, and Professional Writer ». Reading and Writing 23(7), p. 853-888. BADDELEY, A. (1986). Working Memory. Londres : Oxford University Press.

BARBÉRIS, J.-M. \& MAURER, B. (1998). «SUr le "ratage" en discours oral ». L'information grammaticale 77, p. 43-47.

BENVENISTE, É. (1966). «Les relations de temps dans le verbe français ». In : Benveniste, É., Problèmes de linguistique générale. Paris : Gallimard, p. 237-250.

BOURDIN, B. (1999). « Mémoire de travail et production langagière : comparaison de l'oral et de l'écrit chez les adultes et les enfants ». L'année psychologique 99(1), p. 123-148.

BUCHETON, D. (1996). « L'épaississement du texte par la réécriture ». In : David, S \& Plane, S. (dirs), L'apprentissage de l'écriture de l'école au collège. Paris : Presses universitaires de France, p. 159-184. CHANQUOY, L. \& ALAMARGOT, D. (2002). « Mémoire de travail et rédaction de textes : Évolution des modèles et bilan des premiers travaux ». L'année psychologique 102, p. 363-398.

DENHIÈRE, G. \& BAUDET, S. (1987). « Traitement du texte ». In : Rondal, J.-A. \& Thibault, J.-P. (éds), Problèmes de psycholinguistique. Bruxelles : Pierre Mardaga éditeur, p. 43-85.

ECO, U. (1994) [1992]. Les limites de l'interprétation. Trad. de l'italien par M. Bouzaher. Paris : Grasset et Fasquelle.

- (1985) [1979]. Lector in fabula. Trad. de l'italien par M. Bouzaher. Paris : Stock.

FAYOL, M. (1999). Des idées au texte. Psychologie cognitive de la production verbale orale et écrite. Paris : Presses universitaires de France.

FAYOL, M. \& LARGY P. (1992). « Une approche cognitive fonctionnelle de l'orthographe grammaticale. Les erreurs d'accord sujet-verbe chez l'enfant et chez l'adulte ». Langages 95 , p. 80-98.

FENOGLIO, I. (2007) «Du texte avant le texte. Formes génétiques et marques énonciatives de prévisions textualisantes ». Langue française 155, p. 8-34.

FENOGLIO, I. \& CHANQUOY, L. (2007). « Avant-Propos. La notion d'avant-texte : point de rencontre pour une compréhension de l'écriture en acte ». Langue française 155, p. 3-7.

FORTIER, G. \& PRÉFONTAINE, C. (1994). « Pauses, relecture et processus d'écriture ». Revue des sciences de l'éducation 20(2), p. 203-220.

GOSSELIN, L. (2006). « De la distinction entre la dimension temporelle de la modalité et la dimension modale de la temporalité ». Cahiers de Praxématique 47, p. 21-52.

GRÉSILLON, A. (1994). Éléments de critique génétique. Lire les manuscrits modernes. Paris : Presses universitaires de France.

GRIZE, J.-B. (1992). « Résumer, mais pourquoi ? ». In : Charolles, M. \& Petitjean, A. (dirs), L'activité résumante. Metz : Centre d'analyse syntaxique de l'université de Metz, p. 53-61. 
Guillaume, G. (1993) [1929]. Temps et verbe. Théorie des aspects, des modes et des temps. Paris : Honoré Champion.

HAMON, P. (2009). Le signe et la consigne. Essai sur la genèse de l'œuvre en régime naturaliste. Genève : Droz.

HAY, L. (1979). « La critique génétique. Origine et perspectives ». In : Hay, L. (dir.), Essais de Critique Génétique. Paris : Flammarion, p. 227-236.

- (2016). «La critique génétique : une autre approche de l'écriture ? In : Plane, S., Bazerman, C., Rondelli, F., Donahue, C., Applebee, A., Boré, C., Carlino, P., Marquillo Llaruy, M., Rogers, P., Russel, D. (éds), Recherches en écriture : regards pluriels. Metz : Crem/Université de Lorraine, p. 605-618.

HAYES, J. \& FLOWER, L. (1980). « Identifying the organization of writing processes ». In: Gregg, L.W. \& Steinberg, E.R. (dirs), Cognitive Processes in Writing. Hillsdales : Laurence Erlbaum, p. 3-30. HEURLEY, L. (2006). « La révision de texte : l'approche de la psychologie cognitive ». Langages 164, p. 10-25.

JOHNSON-LAIRD, P. (1983). Mental Models: toward a cognitive Science of Language. Cambridge : Cambridge University Press.

KELLOGG, R. T. (1999). « Un modèle de la mémoire de travail dans la rédaction ». In : Piolat, A. \& Pelissier, A. (éds), La rédaction de textes. Approche cognitive. Lausanne : Delachaux et Niestlé, p. 103-136.

KellogG, R. T., Olive, T, \& Piolat, A. (2007). « Verbal and Visual Working Memory in Written Sentence Production ». In : Torrance, M., van Waes, L. \& Galbraith, D. (éds), Writing and cognition: Research and applications. Amsterdam : Elsevier, p. 1-12.

KELLOGG, R. T., TURNER, C., WHITEFORD, A. \& MERTENS A. (2016). « The role of working memory in planning and generating sentences ». Journal of Writing Research 7(3), p. 397-416.

KERVYN, B. \& DUFAYS, J.-L. (2003). « Quels usages du stéréotype dans l'écriture au secondaire?» Pratiques 117-118, p. 208-218.

LAFOURCADE, B., 2008, Traitement des contraintes formelles liées au genre et au médium de production par des scripteurs novices, Thèse de doctorat en sciences du langage : Université Paris-Descartes.

LE BIGOT, N., PASSERAULT, J.-M. \& OLIVE, T. (2011). « Implication of visuospatial and temporal information in memory for word location ». Journal of Cognitive Psychology 23(4), p. 522-530.

LE NY, J.-F. (1989). Science cognitive et compréhension du langage. Paris : Presses universitaires de France.

LEBLAY, C. \& CAPOROSSI G. (éds) (2014). Temps de l'écriture. Enregistrements et représentations. Louvainla-Neuve : Academia Bruylant.

LeIJTEN, M. \& VAN WAES, L. (2006). « Inputlog: New Perspectives on the Logging of On-Line Writing ». Studies in Writing 18, p. 73-94.

LEJEUNE, P. (2005). Signes de vie. Le pacte autobiographique 2. Paris : Seuil.

LITTLETON, E. (1998). « Emerging Cognitive Skills for Writing: Sensitivity to Audience Presence in Five-Through Nine-Year-Olds' Speech ». Cognition and Instruction 16(4), p. 399-430.

Lumbroso, O. (2004). Zola, la plume et le compas. La construction de l'espace dans les Rougon-Macquart d'Émile Zola. Paris : Honoré Champion. 
MATSUHASHI, A. (1981) « Pausing and planning: The tempo of written discourse production ». Research in the Teaching of English 15, p. 113-134.

MCKUTCHEN, D. (1998). « Une théorie de la capacité pour la rédaction : la mémoire de travail dans la composition ». In : Piolat, A. \& Pelissier, A. (éds), La rédaction de textes. Approche cognitive. Neuchatel : Delachaux et Niestlé, p. 183-224.

NOLKE, H. (1999). «Linguistique modulaire : principes méthodologiques et applications ». In : Nolke, H. \& Adam, J.-M. (éds), Approches modulaires : de la langue au discours. Neuchâtel : Delachaux et Niestlé, p. 17-74.

PAVEAU, M.-A. (2006). Les prédiscours. Sens, mémoire, cognition. Paris : Presses de la Sorbonne Nouvelle.

- (2007). « Discours et cognition : les prédiscours entre cadres internes et environnement extérieur ». Corela 6. En ligne : http://corela.revues.org/1550.

PÉTILLON, S. (2006). «Style, critique génétique et modèles rédactionnels : perspectives

linguistiques ». Corpus 5. En ligne : http://corpus.revues.org/473.

PÉTILLON, S. \& GANIER F. (2006). « L'étude de la révision de texte : de la mono- à la pluri-

disciplinarité ». Langages 164, p. 3-9.

PIOLAT, A. \& ROUSSEY, J.-F. (1992). « Rédaction de textes. Éléments de psychologie cognitive ». Langages 106, p. 106-125.

PLANE S., RONDELLI, F. \& VÉNÉRIN, C. (2013). «Variations, fidélité, infidélité : l'écriture de discours rapportés par de jeunes scripteurs ». In : Desoutter, C. \& Meillet, C. (éds), Le discours rapporté : approches linguistiques et perspectives didactiques. Berne : Peter Lang, p. 215-232.

PLANE, S. (2003). « Stratégies de réécriture et gestion des contraintes d'écriture par des élèves de l'école élémentaire : ce que nous apprennent des écrits d'enfants sur l'écriture ». Rivista Italiana di Psicolinguistica Applicata 1, p. 57-77.

Plane, S., AlAmARGot, D., \& LeBRAVE, J.-L. (2010). « Temporalité de l'écriture et rôle du texte produit dans l'activité rédactionnelle ». Langages 177, p. 11-34.

PLANE, S., OliVE, T. \& ALAMARGOT D. (2010). « Pour une approche pluridisciplinaire de la production écrite ». Langages 177, p. 3-5.

RICOEUR, P. (1983). Temps et récit I. Paris : Seuil.

SAUSSURE, L. de (2006). Temps et pertinence. Éléments de pragmatique cognitive du temps. Bruxelles : De Boeck-Duculot.

STRÖMQVIST, S., HOLMQVIST, K., JOHANSSON, V., KARLSSON, H. \& WENGELIN, Å. (2006). « What keystrokelogging can reveal about writing ». Studies in Writing 18, p. 45-72.

VETTERS C. (2004). Temps, aspect et narration. Amsterdam : Rodopi.

WEINRICH, H. (1989). Grammaire textuelle du français. Paris : Didier-Hatier.

\section{NOTES}

1. Accès : http://nerial.free.fr/artelio/artelio/spip_052.html. Alain Ayroles est le scénariste de Garulfo et de De cape et de crocs, bédés drôles et érudites.

2. Les données mentionnées dans cet article ont été collectées par Fabienne Rondelli, Christine Vénérin et l'auteure de cet article. 
3. L'orthographe des textes d'élèves a été conservée dans les citations.

4. Je remercie à cette occasion J. Hayes qui a eu la gentillesse de me signaler les travaux d'E. Littleton (1998).

\section{RÉSUMÉS}

Cet article vise à explorer la manière dont les scripteurs reconstruisent au cours de la production d'écrit une image de leur propre texte. Il s'agit là d'un phénomène dont l'analyse relève de plusieurs champs d'investigation mais qui a été peu étudié bien qu'il participe de la dynamique de l'écriture. Prenant appui sur une expérimentation comportant deux épreuves de rappel, nous recensons quelques éléments attestant la variété de mise en œuvre de ces processus de reconstruction, processus que nous appelons resémantisation. Nous signalons en particulier la concurrence de deux stratégies, l'une focalisée sur la schématisation, l'autre sur la mémorisation des constituants linguistiques et attirons l'attention sur le fait que les souvenirs qu'ont les scripteurs de leur propre texte sont évolutifs.

This article aims at exploring how writers rebuild an image of their own text while they produce it. This is a phenomenon whose analysis involves several fields of investigation but has been studied little even though it is part of the dynamics of writing. For this study, we built an experiment with two recall tests. It appeared that the reconstruction process, that we call " resémantisation", can use different ways. We notice in particular the use of two different strategies, one focused on sketching, the other on language components memory. We also observed how the image that the writers built of their own texts changes over time.

INDEX

Mots-clés : écriture, mémoire, resémantisation, recodage

Keywords : writing, memory, resemantisation, recoding

\section{AUTEUR}

\section{SYLVIE PLANE}

Université Paris-Sorbonne, STIH, EA 4509, F-75005, France 